Lebensmittel -

Verschwendung stoppen, aber wie?

\section{In Kürze}

- Ungefähr ein Drittel aller produzierten Lebensmittel wandert in Österreich in den Müll. Allein Haushalte werfen so bis zu 300 Euro pro Jahr weg.

- Viele Ursachen für Verschwendung sind vermeidbar: Überproduktion, ungeeignete Verpackung und Lagerung, oder irreführende Ablaufdaten.

- Abfälle zu reduzieren spart nicht nur Geld. Es schont Ressourcen und wirkt dem Klimawandel entgegen.

- Was kann man tun? Gesetze zur Nahrungsmittelsicherheit sollten überprüft, Direktvermarktung könnte gefördert und Ablaufdaten vereinfacht werden.

\section{Worum geht es?}

ÖsterreicherInnen werfen jeden fünften Lebensmitteleinkauf in den Müll. Mindestens die Hälfte davon lässt sich vermeiden. Aber auch Landwirtschaft, verarbeitende Betriebe, Supermärkte oder die Gastronomie tragen zum Abfallberg bei. In der gesamten Nahrungsmittelkette, vom Bauern bis zum Teller gehen so ungefähr ein Drittel der produzierten Lebensmittel verloren. Insgesamt sind das $260 \mathrm{~kg}$ Abfall pro Kopf im Jahr. Das liegt zwar im EU-Durchschnitt, trotzdem gelingt es etwa der Tschechischen Republik oder der Slowakei um ein Drittel weniger zu verschwenden. Je höher diese Verluste in jedem Schritt der Nahrungskette, desto größer sind die Auswirkungen auf Umwelt und Wirtschaft

Umweltauswirkungen: Verschwendet man Lebensmittel, so werden Ressourcen wie z.B. Anbaufläche, Wasser oder Energie unnötig vergeudet. Global gesehen verlagern sich Ernährungsgewohnheiten hin zu mehr Konsum von Fleisch und anderen tierischen Produkten. Diese verbrauchen aber in der Produktion viel mehr Ressourcen als pflanzliche Produkte. Zudem wächst die Weltbevölkerung und verlangt nach immer mehr Nahrung. Das und die aufwendige Erzeugung tierischer Produkte setzen Nahrungsversorgung und Weltklima weiter unter Druck. In Europa verursacht derzeit jede Tonne Nahrungsmittelabfall zwei Tonnen Treibhausgase. Verschwendung einzudämmen wirkt also auch dem Klimawandel entgegen.

Ökonomische Folgen: Österreichische Haushalte könnten jährlich bis zu 300 Euro sparen. Die höchsten Verluste entstehen, wenn man originalverpackte Produkte wegwirft, aber auch Fleisch, Fisch, Milch und Eier. Getreideprodukte, Obst und Gemüse machen zwar den Großteil des Abfalls aus, aber weil sie relativ billig sind verursachen sie weniger Kosten. Großes Sparpotenzial, nämlich bis zu 395 Millionen Euro, gibt es auch in der Gastronomie. Betriebe schmeißen zwischen fünf und 45 Prozent des zubereiteten Essens in den Abfall. Auch Produzenten und Händler erleiden Verluste, aber die wenigen verfügbaren Studien beziehen sich vor allem auf Haushalte. Der Ressourcenverbrauch wird meist nicht mit einberechnet. Zudem erhöht weggeworfene Nahrung die weltweite Nachfrage und damit steigen die Weltmarktpreise. Die Kaufkraft von ärmeren Menschen sinkt dadurch, vor allem in Entwicklungsländern.

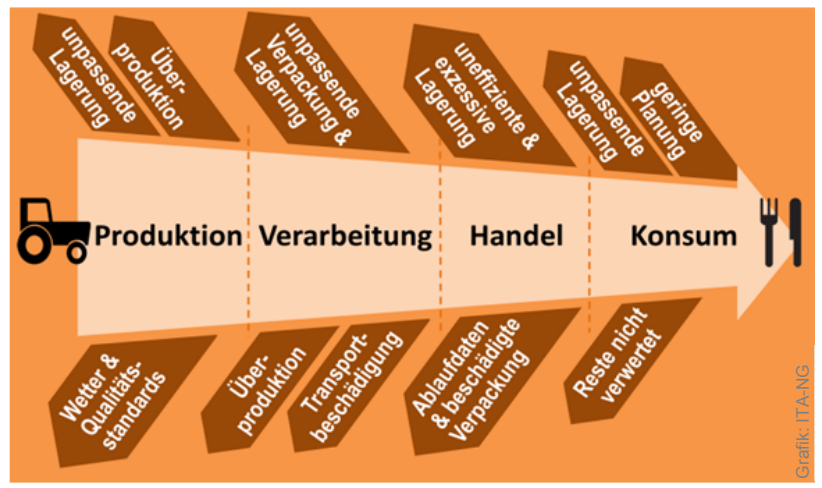

Vom Bauern bis zum Teller: viele Ursachen für Verschwendung.

Warum Verschwendung? KonsumentInnen verursachen fast die Hälfte des gesamten Lebensmittelabfalls in Österreich. Gründe hierfür sind z.B. schlechte Planung beim Einkauf, missverstandene Ablaufdaten, unpassende Lagerung, Zubereiten von zu großen Mahlzeiten oder wenig Bewusstsein für die Verwertung von Resten. In der landwirtschaftlichen Produktion entstehen 30 Prozent des Gesamtabfalls. Schlechtes Wetter, niedrige Marktpreise, die den Ernteaufwand nicht rechtfertigen oder rigoroses Aussortieren aufgrund enger Standards sind die Hauptgründe. Bevor die Ware in den Handel kommt, entstehen 20 Prozent der gesamten Verluste, während des Transports, der Lagerung, der Verarbeitung oder wenn Produkte als ungeeignet aussortiert werden. Der Handel erzeugt kleinere Verluste durch fehlerhafte Verpackung und Etikettierung, abgelaufene Haltbarkeitsdaten oder unzulänglicher Lagerhaltung. 


\section{Ansätze bisher}

Die Europäische Kommission will die Lebensmittelverschwendung bis 2020 halbieren Deshalb vereinfachte sie die Vermarktungsstandards für Obst und Gemüse drastisch. Der Erfolg war gering - in der Praxis wurden kaum mehr Produkte mit abweichende Form, Größe oder Farbe verkauft. Der Grund: Handelsfirmen behielten die alten Standards freiwillig bei, da ihre Logistik (Lagerung, Verpackung, Vertrieb) darauf abgestimmt war. ProduzentInnen, Handel, zivilgesellschaftliche Organisationen und WissenschafterInnen sollten daher neue Standards gemeinsam erstellen. Hier wären Qualitätsmerkmale wie Geschmack, Nährstoffgehalt und Anbaubedingungen zukunftsweisender als Form, Größe oder Farbe.

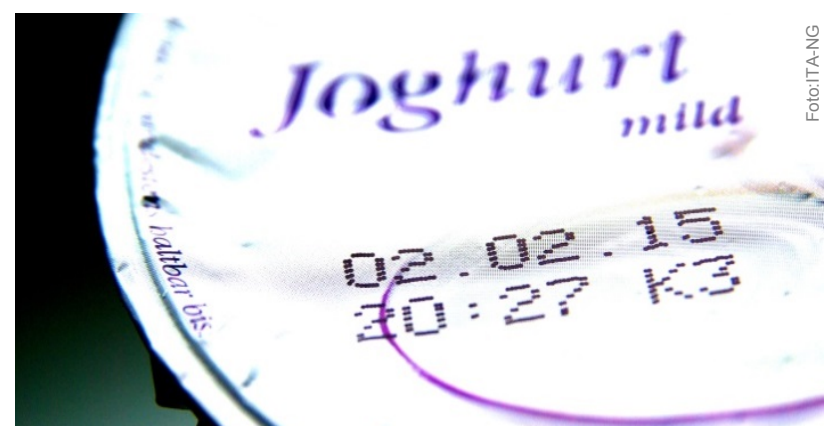

Haushalte verursachen fast die Hälfte des gesamten Lebensmittelabfalls - die Verwechslung von Ablaufdaten ist nur ein Grund

Oft werfen KonsumentInnen original verpackte Produkte mit abgelaufenen Mindesthaltbarkeitsdaten weg. Diese sagen aber nicht direkt etwas über die Nahrungsmittelsicherheit aus und solche Angaben werden auch nicht gesetzlich vorgegeben. Man kann sie eher mit einem Garantiedatum vergleichen, das ProduzentInnen in eigenen Laborstudien ermitteln und sehr eng ansetzen um Haftungen auszuschließen. Im Gegensatz dazu weisen 'zu verbrauchen bis'-Angaben auf den letzten Tag des sicheren Konsums hin. Das ist speziell für leicht verderbliche Waren wie faschiertes Fleisch oder rohen Fisch wichtig. Diese Daten werden sehr häufig verwechselt. Um dem entgegenzuwirken, ließe sich die Etikettierung vereinfachen, die KonsumentInneninformationen und Lesbarkeit verbessern. Auch könnte man Ablaufdaten für lang haltbare Produkte wie Reis oder Nudeln ganz abschaffen.

Der Handel bemüht sich schon jetzt mithilfe neuer Technik wie intelligente Bestellungssysteme um höhere Effizienz. RFID-Chips messen beispielsweise Temperaturdaten während des Transportes. Auch andere Technologien sollen Lebensmittelverluste an verschiedenen Stellen der Nahrungskette verringern, etwa, intelligente' Etiketten, Kühlschränke, Einkaufswagen oder Mülleimer. Allerdings werden diese gerade erst entwickelt. Es ist bisher nicht absehbar wieviel sie wirklich zur Lösung des Problems beitragen, welche Nebeneffekte sie haben und ob es nicht zu unvorhergesehenen Folgen (Rebound) kommt.

\section{Was tun?}

Die Nahrungsversorgung ist längst globalisiert. Lebensmittelabfälle zu reduzieren ist deshalb eine internationale Aufgabe. Trotzdem kann Österreich unmittelbare Chancen nutzen um den stärksten Treibern zu begegnen.

- Die jetzigen Gesetze zur Nahrungsmittelsicherheit sollten dahingehend überprüft werden, welche Bestimmungen zwar viel Abfall hervorrufen, aber für den Schutz der Gesundheit nicht zwingend erforderlich sind.

- Vermarktungsstandards könnten abgeändert werden: vom bloßen Aussehen hin zu Verzehrqualitäten, wie Geschmack, Nährstoffgehalt und Anbaubedingungen.

- $\quad$ Direkte Vermarktung von Obst und Gemüse, das den gängigen Standards nicht entspricht, würde Abfälle und Transportdistanzen verringern. Bauernmärkte, Produktionskooperativen, solidarische Einkaufsgemeinschaften oder Gemeinschaftshöfe (community supported agriculture) sind einige Möglichkeiten.

- Verbesserte Haltbarkeitsdaten sollten die echte Lagerfähigkeit von Produkten beschreiben. Das könnte Verwechselungen von Mindesthaltbarkeit und 'zu verbrauchen bis' entgegenwirken. Ablaufdaten für lang haltbare Produkte könnten abgeschafft werden.

- $\quad$ Eine Haftungsbegrenzung für Spenden- und Wohltätigkeitsorganisationen sowie die Förderung von privaten Initiativen zur Verteilung von überschüssigen Lebensmitteln (food-sharing) kann viele Abfälle vermeiden und ökonomisch benachteiligten Menschen helfen.

\section{Zum Weiterlesen}

Priefer, C.; Jörissen, J.; Bräutigam, K.-R.: Technology options for feeding 10 billion people. Options for cutting food waste - Final report. Brüssel, Belgien: European Parliament 2013 (STOA - Science and Technology Options Assessment / ETAG) www.europarl.europa.eu/RegData/etudes/etudes/ioin/2013/513515/IP OL-JOIN ET\%282013\%29513515 EN.pdf

\section{Kontakt}

Mag. Niklas Gudowsky

E-mail: tamail@oeaw.ac.at

Telefon: $+43(1) 51581-6582$

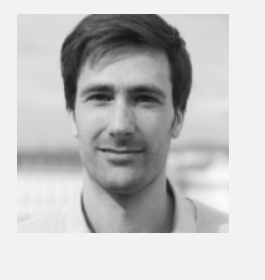

IMPRESSUM: Medieninhaber: Österreichische Akademie der Wissenschaften; Juristische Person öffentlichen Rechts (BGBI. 569/1921 i.d.F. BGBI. I 130/2003); Dr. Ignaz Seipel-Platz 2, A-1010 Wien; Herausgeber: Institut für Technikfolgen-Abschätzung (ITA); Strohgasse 45/5, A-1030 Wien; www. oeaw.ac.at/ita | Erscheinungsweise: Die ITA-Dossiers erscheinen unregelmäßig und dienen der Veröffentlichung der Forschungsergebnisse des ITA. Die Dossiers werden in kleiner Auflage gedruckt und open access über das Portal „epub.oeaw“ zur Verfügung gestellt: epub.oeaw.ac.at/ita/ita-dossiers | ISSN: 2306-1960 Dieses Werk ist lizenziert unter einer Creative Commons Namensnennung 4.0 International Lizenz: creativecommons org/licenses/by/4 O/deed de ITA-Dossier Nr. 014, März 2015, epub. oeaw. ac. at/ita/ita-dossiers/ita-dossier014.pdf | Koautor: H. Torgersen 